This is the final peer-reviewed accepted manuscript of:

Delorme, M., lori, M. \& Martello, S. BPPLIB: a library for bin packing and cutting stock problems. Optim Lett 12, 235-250 (2018).

The final published version is available online at:

https://doi-org.ezproxy.unibo.it/10.1007/s11590-017-1192-z

Rights / License:

The terms and conditions for the reuse of this version of the manuscript are specified in the publishing policy. For all terms of use and more information see the publisher's website. 
1

\title{
BPPLIB: A Library for Bin Packing and Cutting Stock Problems
}

\author{
Maxence Delorme ${ }^{(1)}$, Manuel Iori ${ }^{(2)}$, Silvano Martello ${ }^{(1)}$ \\ (1) DEI "Guglielmo Marconi", University of Bologna \\ (2) DISMI, University of Modena and Reggio Emilia \\ maxence.delorme2@unibo.it, manuel.iori@unimore.it, silvano.martello@unibo.it
}

\begin{abstract}
The bin packing problem (and its variant, the cutting stock problem) is among the most intensively studied combinatorial optimization problems. We present a library of computer codes, benchmark instances, and pointers to relevant articles for these two problems. The library is available at http://or.dei.unibo.it/library/bpplib. The computer code section includes twelve programs: seven are directly downloadable from the library page, while for the remaining five we provide addresses where they can be obtained or downloaded. Some of the codes for which we provide an original $\mathrm{C}++$ implementation need an integer linear programming solver. For such cases, the library provides two versions: one that uses the commercial solver CPLEX, and one that uses the freeware solver SCIP. The benchmark section provides over six thousands instances (partly coming from the literature and partly randomly generated), together with the corresponding solutions. Instances that are difficult to solve to proven optimality are included. The library also includes a BibTeX file of more than 150 references on this topic and an interactive visual tool to manually solve bin packing and cutting stock instances. We conclude this work by reporting the results of new computational experiments on a number of computer codes and benchmark instances.
\end{abstract}

Keywords: Bin packing, Cutting stock, Computer codes, Benchmark instances, Surveys.

\section{Introduction}

In the bin packing problem (BPP), $n$ items of given integer weight $w_{j}(j=1, \ldots, n)$ have to be packed into the minimum number of identical containers (bins) of integer capacity c. Let $u$ be any upper bound on the solution value. Let us introduce two sets of binary variables: $y_{i}(i=1, \ldots, u)$, taking the value one if and only if bin $i$ is used in the solution, and $x_{i j}$ $(i=1, \ldots, u ; j=1, \ldots, n)$, taking the value one if and only if item $j$ is packed into bin $i$. A possible simple Integer Linear Programming (ILP) model of the problem is then (see Martello and Toth [28])

$$
\begin{array}{lll}
\min & \sum_{i=1}^{u} y_{i} & \\
\text { s.t. } & \sum_{j=1}^{n} w_{j} x_{i j} \leq c y_{i} & (i=1, \ldots, u), \\
& \sum_{i=1}^{u} x_{i j}=1 & (j=1, \ldots, n), \\
& y_{i} \in\{0,1\} & (i=1, \ldots, u), \\
& x_{i j} \in\{0,1\} & (i=1, \ldots, u ; j=1, \ldots, n) .
\end{array}
$$


The BPP is known to be $\mathcal{N} \mathcal{P}$-hard in the strong sense (by transformation from the 3Partition problem, see Garey and Johnson [20]). As any instance of either problem can easily be transformed into an equivalent instance of the other, the same holds for the CSP.

These two problems are among the most intensively studied problems in combinatorial optimization. Two recent surveys on exact methods (Delorme, Iori, and Martello [14]) and approximation algorithms (Coffman, Csirik, Galambos, Martello, and Vigo [10]) consider in total over 230 different references. Previous surveys were presented by Garey and Johnson [21], Coffman, Garey, and Johnson [11, 12], Sweeney and Paternoster [35], Dyckhoff [16], Martello and Toth [28] (Chapter 8), Dyckhoff and Finke [17], Valério de Carvalho [38], Wäscher, Haußner, and Schumann [40], among others. Most solution methodologies have been tried on these problems: different kinds of ILP models, lower bound computations, branch-and-bound, branch-and-price, constraint programming, approximation algorithms, heuristics, and metaheuristics.

In the next section we list a number of relevant web-based libraries for optimization problems. In Section 3 we introduce the computer codes and the visual solver provided by the BPPLIB. In Section 4 we describe the available benchmarks: some of them were used in [14] for the computational evaluations of the different exact approaches, using commercial solver CPLEX when needed. As the library has been enriched by also providing versions based on the freeware solver SCIP, in Section 5 we provide new experiments aiming at evaluating the computational difference between the two versions. In addition, we describe new test instances, that appeared after the publication of [14], and present the corresponding computational experiments.

\section{Web-based libraries for optimization problems}

A number of web-based libraries dedicated to different optimization problems can be found on the Internet. The oldest one is probably the famous 

Assignment Problem, see http://anjos.mgi.polymtl.ca/qaplib/. It provides instances, relevant references, and computer codes;

- Traveling Salesman Problem web page, created by Applegate, Bixby, Chvatal, and Cook [1], see http://www.math. uwaterloo.ca/tsp/. It contains instances, computer codes, pointers to the literature, and educational tools;

- VRPH, a library of heuristics for the Vehicle Routing Problems (VRP), authored by Groër, Golden, and Wasil [23], see http://sites.google.com/site/vrphlibrary/;

- MIPLIB, a Mixed Integer Programming library, providing a large set of instances, created by Koch et al. [25], see http://miplib.zib.de/;

- libcgrpp, a library for Bound-Constrained Global Optimization, implemented by Silva, Resende, Pardalos, and Hirsch [34], see http://www.swmath.org/software/7205, containing a number of computer codes;

- CBLIB 2014, a collection of benchmark problems for Conic Mixed-Integer and Continuous Optimization, constructed by Friberg [19], see http://cblib.zib.de/;

- CVRPLIB, a repository of VRP benchmark instances and solutions, designed by Uchoa et al. [36], see http://vrp.atd-lab.inf.puc-rio.br/. It also proposes rewards for the solution of challenging instances.

We present here the BPPLIB, a library dedicated to Bin Packing and Cutting Stock Problems, available at http://or.dei.unibo.it/library/bpplib. An earlier, smaller version of the library was implemented as an auxiliary instrument for the computational experiments presented in [14]. The current BPPLIB contains pointers to the literature, original computer codes, links to computer codes from the Internet, benchmark instances, and an open source visual application to interactively solve BPP instances. Data sets and problem generators for the BPP and the CSP can also be found at the page of ESICUP (the EURO working group on cutting and packing), https://paginas.fe.up.pt/ esicup/about.

\section{Computer codes}

The BPPLIB provides twelve computer codes of different types for the exact solution of the BPP and the CSP. The choice of such codes was motivated by a number of properties: historical relevance, efficiency, reliability, and availability of the corresponding computer codes. The main characteristics of the codes are summarized in Table 1. More detailed information is provided below. 
Table 1: Main characteristics of the computer codes provided by the BPPLIB.

\begin{tabular}{|c|c|c|c|c|c|c|c|c|c|c|}
\hline \multirow{2}{*}{ Code } & \multirow{2}{*}{ Problem } & \multirow{2}{*}{ BPPLIB } & \multirow{2}{*}{ Language } & \multirow{2}{*}{ Type } & \multirow{2}{*}{ Ref. } & \multicolumn{2}{|l|}{ Computer code } & \multicolumn{3}{|c|}{ Supported ILP solver } \\
\hline & & & & & & Author & Year & CPLEX & Gurobi & Scip \\
\hline MTP & BPP & code & Fortran & $B \& B$ & [28] & Martello and Toth & 1990 & & required & \\
\hline BISON & BPP & pointer & Pascal & $B \& B$ & [32] & Scholl, Klein, and Jürgens & 1997 & not & required & \\
\hline CVRPSEP & $\mathrm{BPP}$ & link & $\mathrm{C}$ & B\&B & {$[27]$} & Lysgaard & 2004 & not & required & \\
\hline BELOV & CSP & link & $\mathrm{C}++$ & $\mathrm{B} \& \mathrm{C} \& \mathrm{P}$ & {$[3]$} & Belov & 2006 & $\mathrm{x}$ & & \\
\hline SCIP-BP & BPP & link & $\mathrm{C}$ & $\mathrm{B} \& \mathrm{P}$ & {$[30]$} & Berthold and Heinz & c. 2010 & & & $\mathrm{x}$ \\
\hline ONECUT & CSP & code & $\mathrm{C}++$ & ILP & {$[15]$} & Delorme & 2014 & $\mathrm{x}$ & & $\mathrm{x}$ \\
\hline ARCFLOW & CSP & code & $\mathrm{C}++$ & ILP & [37] & Delorme & 2014 & $\mathrm{x}$ & & $\mathrm{x}$ \\
\hline DPFLOW & $\mathrm{BPP}$ & code & $\mathrm{C}++$ & ILP & {$[8]$} & Delorme & 2014 & $\mathrm{x}$ & & $\mathrm{x}$ \\
\hline VPSOLVER & CSP & link & $\mathrm{C}++$ & ILP & {$[5]$} & Brandão & 2014 & $\mathrm{x}$ & $\mathrm{x}$ & $\mathrm{x}$ \\
\hline
\end{tabular}

\section{Branch-and-bound}

Implicit enumeration has been the first tool for the study of methods for optimally solving the BPP. The first effective exact algorithms for the BPP were indeed based on a branchand-bound approach. The library provides, in chronological order:

- MTP: Fortran code of the BPP algorithm by Martello and Toth [28], originally available in the diskette accompanying the book. The algorithm adopts a depth-first strategy to explore a branch-decision tree that considers one item per level: descendant nodes are generated by assigning the current item, in turn, to all already initialized bins and possibly to a new bin. While the approach is effective for BPP instances, considering one item at a time is clearly inefficient for CSP instances with high item multiplicity. The code can be run using the Fortran front end of the GNU Compiler Collection GCC;

- BISON: Scholl, Klein, and Jürgens [32] obtained a very efficient BPP algorithm by enriching MTP through new lower bounds and a Tabu search algorithm to help the search by means of effective heuristic solutions. The code was implemented in Pascal, and can be obtained from the authors, using the address provided in the library. Worth is mentioning that, in spite of its 'age', this program is still working and quite effective: at the time of writing, it can be run using compiler fpc (version 3.0.0 for x86_64);

- CVRPSEP: we provide a link to the C code implemented by J. Lysgaard as part of a separation routine within the algorithm by Lysgaard, Letchford, and Eglese [27] for the capacitated vehicle routing problem. The routine was obtained by using procedures from MTP. It is generally less efficient than MTP, but we decided to include it in the library mainly because one may prefer a $\mathrm{C}$ code to a Fortran code. The implementation details can be found in a technical report by Lysgaard [26].

\section{Branch-and-price}

Branch-and-price is the modern evolution of branch-and-bound, obtained by combining implicit enumeration and column generation. The resulting approach can produce very effective algorithms for the problems at hand. We provide links to two computer codes: 


\section{Pseudo-polynomial formulations solved via ILP}

Already in the Seventies, pseudo-polynomial models coming from a graph representation of the solution space were proposed. For many years, solution approaches based on such models have been regarded as very theoretical, with no practical interest, due to the huge number of variables and constraints they imply. Up to few years ago, these methods were mainly used within branch-and-price algorithms (see, e.g., Valério de Carvalho [37], who proposed to solve his pseudo-polynomial model through column generation). However, nowadays computational power of ILP solvers made them competitive with branch-and-price algorithm also for the case of realistic size instances, provided the number of generated variables (that depends on capacity, number of items, and item weights) is not too big. The BPPLIB provides four algorithms based on pseudo-polynomial models:

- ONECUT: C++ implementation of the one-cut CSP model independently defined in the Seventies by Rao [29], and Dyckhoff [15];

- ARCFLOW: C++ implementation of the arc-flow CSP model by Valério de Carvalho [37];

- DPFLOW: $\mathrm{C}++$ implementation of the DP-flow BPP model by Cambazard and O'Sullivan [8];

- VPSOLVER: link to the $\mathrm{C}++$ implementation by Brandão and Pedroso [5] of their CSP algorithm. This is currently the most effective pseudo-polynomial approach, and its performance is often competitive with that of BELOV.

For the first three codes we provide both a version that uses CPLEX as an inner routine, and a version that uses SCIP, together with the corresponding makefiles. Code VPSOLVER was instead implemented by the authors in a version that invokes Gurobi.

\section{BppGame: An interactive visual solver}

The library includes the pointer to an open source visual ScalaFX application to interactively solve BPP and CSP instances. The application is derived from a more general tool for the solution of two-dimensional packing problems, see Costa, Delorme, Iori, Malaguti, and Martello [13]. It allows an easy interaction to obtain a feasible solution of a given problem instance. The application has a number of features, that are fully described in its own web page http://gianlucacosta.info/BppGame/. The easiest way to test it consists in following the hyperlink and executing the sequence of actions: Download zip and extract 
its contents $\rightarrow$ BppGame-x.x $\rightarrow$ bin $\rightarrow$ BppGame.bat (Windows) or BppGame (Linux) $\rightarrow$ Sample problems. Figure 1 shows a BppGame visualization. The user can click on an item on the right frame, and drag and drop it to a selected position in the left frame.

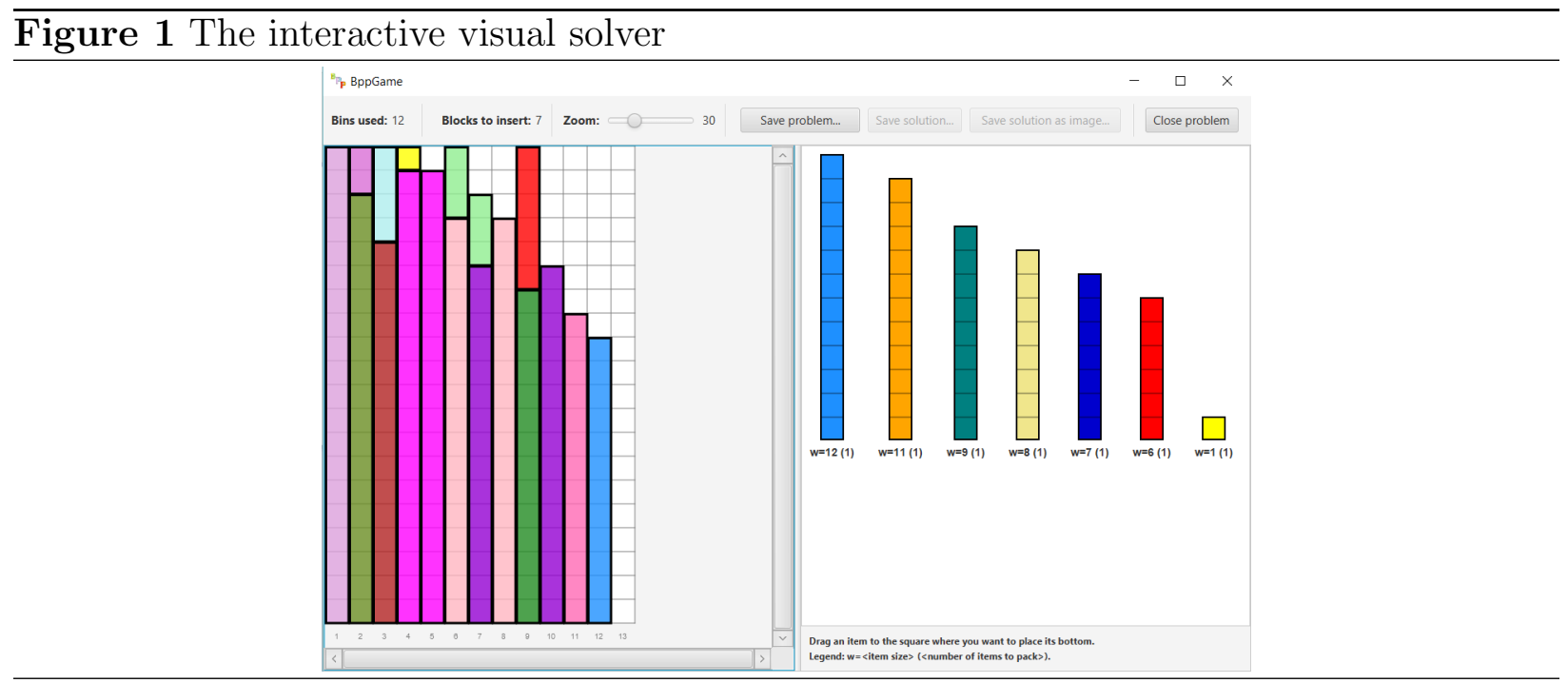

\section{Benchmarks}

The BPPLIB provides 6195 benchmark instances belonging to four categories. Each instance is provided, using unified formats, both in BPP and CSP version. The main characteristics of the instances are summarized in Table 2. Detailed information is provided below.

Table 2: Main characteristics of the instances provided by the BPPLIB.

\begin{tabular}{|c|c|c|c|c|c|c|c|c|}
\hline \multirow{2}{*}{ Instances } & \multirow{2}{*}{ Ref. } & \multicolumn{4}{|c|}{ Parameters of the instances } & \multicolumn{3}{|c|}{ Specificity } \\
\hline & & \# & $n$ & $c$ & Distribution & Large $c$ & $c$ Perf. pack. & Non-IRUP \\
\hline Falkenauer U & [18] & 80 & $\{120,250,500,1000\}$ & 150 & uniform & & & \\
\hline Falkenauer $\mathrm{T}$ & {$[18]$} & 80 & $\{60,120,249,501\}$ & 1000 & ad-hoc & & $\mathrm{x}$ & \\
\hline Scholl1 & {$[32]$} & 720 & $\{50,100,200,500\}$ & $\{100,120,150\}$ & uniform & & & \\
\hline Scholl2 & {$[32]$} & 480 & $\{50,100,200,500\}$ & 1000 & uniform & & & \\
\hline Scholl3 & {$[32]$} & 10 & 200 & 100000 & uniform & $\mathrm{x}$ & & \\
\hline Wascher & {$[39]$} & 17 & {$[57-239]$} & 10000 & ad-hoc & $\mathrm{x}$ & $\mathrm{x}$ & $\mathrm{x}$ \\
\hline Schwerin1 & [33] & 100 & 100 & 1000 & uniform & & & \\
\hline Schwerin2 & [33] & 100 & 120 & 1000 & uniform & & & \\
\hline $\operatorname{Hard} 28$ & {$[31]$} & 28 & $\{160,180,200\}$ & 1000 & ad-hoc & & $\mathrm{x}$ & $\mathrm{x}$ \\
\hline Random & {$[14]$} & 3840 & $\{50,100,200 \ldots 500,750,1000\}$ & $\{50,75,100,120 \ldots 750,1000\}$ & uniform & & & \\
\hline $\mathrm{AI}$ & [14] & 250 & $\{202,403,601,802,1003\}$ & $\leq\{2500,10 \mathrm{~K}, 20 \mathrm{~K}, 40 \mathrm{~K}, 80 \mathrm{~K}\}$ & ad-hoc & $\mathrm{x}$ & $\mathrm{x}$ & \\
\hline ANI & {$[14]$} & 250 & $\{201,402,600,801,1002\}$ & $\leq\{2500,10 \mathrm{~K}, 20 \mathrm{~K}, 40 \mathrm{~K}, 80 \mathrm{~K}\}$ & ad-hoc & $\mathrm{x}$ & & $\mathrm{x}$ \\
\hline GI & [24] & 240 & $\sim 10.5 \times\{125,250,500\}$ & $\{500000,1500000\}$ & uniform & $\mathrm{x}$ & & \\
\hline
\end{tabular}

\section{Literature instances}

This section contains the 1615 instances proposed by

- Falkenauer [18]: 80 (easy) instances with uniformly distributed item sizes and 80 (more difficult) instances obtained through triplets of items that, in any optimal solution, must be packed into the same bin wihtout leaving unused space (perfect packing). 
- Scholl, Klein, and Jürgens [32]: three sets of instances with uniformly distributed item sizes. The first set is composed by 720 easy instances, the second set by 480 instances of medium difficulty, and the third set by 10 difficult instances characterized by huge capacities;

- Wäscher and Gau [39]: 17 very hard instances selected by the authors from a much larger set of instances belonging to different typologies;

- Schwerin and Wäscher [33]: two sets of 100 relatively easy instances each;

- Schoenfield [31]: 28 hard instances that do not involve huge capacities.

In the library, each set is identified by the name of the (first) author. Additional instances can be created through the provided link to the instance generators proposed by Schwerin and Wäscher [33] and Gau and G. Wäscher [22].

\section{Randomly generated instances}

The library provides the 3840 instances that were randomly generated for the computational experiments reported in [14]. The instances have different values of $n(50,100,200,300,400$, $500,750,1000)$, of $c(50,75,100,120,125,150,200,300,400,500,750,1000)$, and of the minimum $(0.1 c, 0.2 c)$ and maximum $(0.7 c, 0.8 c)$ item weight. The benchmark contains 10 instances for each of the 384 quadruplets ( $n, c$, minimum weight, maximum weight). These instances are relatively easy, and the algorithms listed in Section 3 could solve most of them within reasonable CPU times.

\section{Hard instances}

An instance of an optimization problem that possesses the so-called Integer Round-Up Property (see Berge and Johnson [4]) is called an IRUP instance and is generally considered less difficult to solve in practice with respect to instances not possessing such property. In order to perform experiments on challenging instances, a number of so called augmented Non-IRUP and augmented IRUP instances were proposed in [14], using as a basis a set of Non-IRUP instances presented in Caprara, Dell'Amico, Díaz Díaz, Iori, and Rizzi [9]. For the 250 instances of the former class an optimal solution is easy to find, but its optimality is very difficult to prove. Even the continuous relaxation of the set covering formulation (the basis of branch-and-price algorithms) and that of the pseudo-polynomial formulations fail in reaching the optimal value. As a consequence, algorithms based on such relaxations require either a huge branching process or a heavy cut generation: already for $n \approx 400$, no algorithm is capable of solving all of them to proven optimality. For the 250 instances of the latter class, it is easy to produce a lower bound whose value is equal to the optimum, but it is difficult to build an optimal solution.

\section{GI instances}

The library includes 240 new instances, recently proposed by Gschwind and Irnich [24]. Such instances, uniformly randomly generated, are characterized by very large capacities. They are organized into four sets of 60 instances each. As shown in the next section, two of such sets are generally difficult to solve. 
Table 4: Benchmarks from the literature (non-trivial instances only), pseudo-polynomial models. Number of instances solved in less than one minute (average CPU time in seconds).

\begin{tabular}{|c|c|c|c|c|c|c|c|c|}
\hline \multirow{2}{*}{ Set } & \multirow{2}{*}{$\begin{array}{l}\text { Number } \\
\text { of tested } \\
\text { instances }\end{array}$} & \multicolumn{2}{|c|}{ ONECUT } & \multicolumn{2}{|c|}{ ARCFLOW } & \multicolumn{2}{|c|}{ DPFLOW } & \multirow{2}{*}{ VPSOLVER } \\
\hline & & CPLEX & SCIP & CPLEX & SCIP & CPLEX & SCIP & \\
\hline Falkenauer U & 74 & $\mathbf{7 4}(0.2)$ & $67(23.8)$ & $\mathbf{7 4}(0.2)$ & $70(18.7)$ & $37(38.8)$ & $0(60.0)$ & $\mathbf{7 4}(0.1)$ \\
\hline Falkenauer $\mathrm{T}$ & 80 & $80 \quad(8.7)$ & $21(44.9)$ & 80 & $33(41.4)$ & $40(41.7)$ & $20(50.8)$ & $80 \quad(0.4)$ \\
\hline Scholl1 & 323 & $323 \quad(0.1)$ & $318 \quad(5.0)$ & $323 \quad(0.1)$ & $320 \quad(5.1)$ & $289(13.0)$ & $178(34.0)$ & $\mathbf{3 2 3}(0.1)$ \\
\hline Scholl2 & 244 & $118(38.7)$ & $20(56.3)$ & $202(18.9)$ & $39(53.7)$ & $58(50.4)$ & $11(58.5)$ & $208(14.0)$ \\
\hline Scholl3 & 10 & $0(60.0)$ & $0(60.0)$ & $0(60.0)$ & $0(60.0)$ & $0(60.0)$ & $0(60.0)$ & $10(6.3)$ \\
\hline Schwerin1 & 100 & $100(13.1)$ & $0(60.0)$ & $100(1.5)$ & $0(60.0)$ & $0(60.0)$ & $0(60.0)$ & $100(0.3)$ \\
\hline Schwerin2 & 100 & $100(11.7)$ & $0(60.0)$ & 100 & $1(59.5)$ & $0(60.0)$ & $0(60.0)$ & 100 \\
\hline Hard28 & 28 & $6(54.6)$ & $0(60.0)$ & $16(40.6)$ & $0(60.0)$ & $0(60.0)$ & $0(60.0)$ & $27(14.2)$ \\
\hline Total (average) & 976 & $801(16.3)$ & $426(36.9)$ & $895(8.2)$ & $463(35.6)$ & $424(38.9)$ & $209(50.3)$ & 928 \\
\hline
\end{tabular}


Table 4 shows in addition that the performance of ONECUT and ARCFLOW is not affected by the ILP solver for most of the easy instances, while their performance for the difficult instances sharply worsens when SCIP is used instead of CPLEX. This behavior could be explained by the difference in the number of generated variables and constraints between different benchmarks. For example, ARCFLOW produces, on average, 1735 variables and 103 constraints for Scholl 1 instances, while for "Scholl 2" it produces, on average, 39307 variables and 840 constraints.

Tables 5 and 6 refer to the randomly generated instances and provide the same information as in Tables 3 and 4. The previous observations are confirmed: BELOV and VPSOLVER outperform the other approaches, and the use of SCIP decreases the algorithms' performance, especially for large values of $n$.

Table 5: Randomly generated benchmarks (non-trivial instances only), enumerative algorithms. Number of instances solved in less than one minute (average CPU time in seconds).

\begin{tabular}{|c|c|c|c|c|c|c|}
\hline \multirow{2}{*}{$n$} & \multirow{2}{*}{$\begin{array}{l}\text { Number } \\
\text { of tested } \\
\text { instances }\end{array}$} & \multicolumn{3}{|c|}{ Branch-and-bound } & \multicolumn{2}{|c|}{ Branch-and-price } \\
\hline & & MTP & BISON & CVRPSEP & BELOV & SCIP-BP \\
\hline 50 & 165 & $(0.8)$ & $(0.0)$ & $(0.4)$ & $165(0.0)$ & 165 \\
\hline 100 & 271 & $243 \quad(7.4)$ & $(3.8)$ & $(8.4)$ & $271(0.0)$ & 271 \\
\hline 200 & 359 & $237(21.6)$ & $290(12.0)$ & $220(25.0)$ & $359(0.0)$ & $293(22.6)$ \\
\hline 300 & 393 & $166(35.7)$ & $265(20.7)$ & $144(38.7)$ & $393(0.1)$ & $155(44.1)$ \\
\hline 400 & 425 & $151(39.1)$ & $244(26.1)$ & $138(41.2)$ & $425(0.2)$ & $114(49.8)$ \\
\hline 500 & 414 & $121(43.0)$ & $208(30.3)$ & $128(42.6)$ & $414(0.2)$ & $69(55.1)$ \\
\hline 750 & 433 & $93(47.3)$ & $214(30.9)$ & $98(47.3)$ & $433(0.4)$ & $22(59.5)$ \\
\hline 1000 & 441 & $78(49.5)$ & $196(33.9)$ & $73(50.8)$ & $441(0.7)$ & $0(60.0)$ \\
\hline Total (average) & 2901 & $1252(34.7)$ & $1839(22.6)$ & $1204(36.0)$ & $2901(0.2)$ & $1089(42.4)$ \\
\hline
\end{tabular}

Table 6: Randomly generated benchmarks (non-trivial instances only), pseudo-polynomial models. Number of instances solved in less than one minute (average CPU time in seconds).

\begin{tabular}{|c|c|c|c|c|c|c|c|c|}
\hline \multirow{2}{*}{$n$} & \multirow{2}{*}{$\begin{array}{l}\text { Number } \\
\text { of tested } \\
\text { instances }\end{array}$} & \multicolumn{2}{|c|}{ ONECUT } & \multicolumn{2}{|c|}{ ARCFLOW } & \multicolumn{2}{|c|}{ DPFLOW } & \multirow{2}{*}{ VPSOLVER } \\
\hline & & CPLEX & SCIP & CPLEX & SCIP & CPLEX & SCIP & \\
\hline 50 & 165 & $165(0.1)$ & $163(2.0)$ & $165(0.1)$ & 165 (1.6) & $165(0.5)$ & $162 \quad(5.1)$ & $165(0.0)$ \\
\hline 100 & 271 & $271(0.8)$ & $249 \quad(8.6)$ & $271(0.3)$ & $262(10.1)$ & 271 & $168(34.5)$ & $271(0.1)$ \\
\hline 200 & 359 & $358(2.4)$ & $286(15.4)$ & $359(0.8)$ & $278(20.2)$ & $292(21.0)$ & $76(51.8)$ & $359(0.3)$ \\
\hline 300 & 393 & $385(4.5)$ & $272(22.2)$ & $391(2.0)$ & $262(24.9)$ & $243(33.9)$ & $31(57.3)$ & $393(0.6)$ \\
\hline 400 & 425 & $408(5.1)$ & $293(22.0)$ & $421(3.0)$ & $276(25.8)$ & $193(42.4)$ & $23(58.1)$ & $425(0.8)$ \\
\hline 500 & 414 & $394(6.3)$ & $275(24.0)$ & $402(4.0)$ & $258(26.5)$ & $169(44.8)$ & $13(58.8)$ & $413(1.7)$ \\
\hline 750 & 433 & $401(7.8)$ & $284(24.3)$ & $415(6.0)$ & $279(25.7)$ & $120(52.6)$ & $12(59.1)$ & $431(2.4)$ \\
\hline 1000 & 441 & $407(8.1)$ & $280(25.8)$ & $416(6.8)$ & $281(26.1)$ & $67(56.4)$ & $7(59.6)$ & $434(3.4)$ \\
\hline Total (average) & 2901 & $2789(5.0)$ & $2102(20.0)$ & $2840(3.3)$ & $2061(22.3)$ & $1520(36.7)$ & $492(52.5)$ & 2891 (1.4) \\
\hline
\end{tabular}

We report in Table 7 the results of computational experiments for the GI benchmark, a set of CSP instances recently proposed by Gschwind and Irnich [24] for testing their dual inequalities aimed at stabilizing column generation processes. They are organized into four groups (AA, AB, BA, and BB), characterized by different item weight ranges and capacities. Each group has three sets of 20 instances each, characterized by the number of item types $(125,250$, and 500). 
We tested the best enumerative algorithm (BELOV) and the best pseudo-polynomial approaches (ARCFLOW and VPSOLVER) with a time limit of one hour. BELOV could solve all of these instances very quickly, while they turned out to be extremely difficult for the pseudo-polynomial models. The behavior of the latter approaches was particularly poor for the instances that have items with very small weight and huge capacities (AB and BB, with $c \geq 500000$ ), which induce a high number of variables and constraints. For example, the ILP model produced by ARCFLOW has on average 549441 variables and 131219 constraints for instances AA with $m=125$, but 5754617 variables and 404283 constraints for instances AB with $m=125$.

\section{Conclusions and future research}

We presented the BPPLIB, a library dedicated to Bin Packing and Cutting Stock Problems that provides computer codes, benchmark instances, pointers to relevant research papers, a bibliography, and an interactive visual solver. The computer code section includes twelve exact programs that make use of different optimization paradigms. To fit the needs of both researchers and practitioners, we provide two versions of the programs that need an ILP solver: one using the commercial solver CPLEX and the other using the freeware solver SCIP. The benchmark section provides over six thousands instances, among which a few hundreds remain unsolved to proven optimality despite the application of all available exact methods.

We believe that the BPPLIB can be a useful tool to foster new research on the challenging area of Bin Packing and Cutting Stock optimization. In addition, it can be used for educational purposes as it provides an interactive tool (the "BppGame", see Figure 1) that students can use to easily understand the features and the difficulty of optimization problems.

As future activity, we plan to maintain the library updated with new relevant contributions that will appear on these problems, as well as to expand it through the most interesting problem variants. 


\section{Acknowledgements}

Research supported by Air Force Office of Scientific Research (Grant FA9550-17-1-0067) and by MIUR-Italy (Grant PRIN 2015). We thank Gianluca Costa for the development of the BppGame. We thank the reviewers for useful comments.

\section{References}

[1] D.L. Applegate, R.E. Bixby, V. Chvatal, and W.J. Cook. The Traveling Salesman Problem - A Computational Study. Princeton University Press, Princeton, NJ, 2006.

[2] J. E. Beasley. OR-library: distributing test problems by electronic mail. Journal of the Operational Research Society, 41:1069-1072, 1990.

[3] G. Belov and G. Scheithauer. A branch-and-cut-and-price algorithm for one-dimensional stock cutting and two-dimensional two-stage cutting. European Journal of Operational Research, 171:85-106, 2006.

[4] C. Berge and E.L. Johnson. Coloring the edges of a hypergraph and linear programming techniques. Annals of Discrete Mathematics, 1:65-78, 1977.

[5] F. Brandão and J.P. Pedroso. Bin packing and related problems: General arc-flow formulation with graph compression. Computers \& Operations Research, 69:56-67, 2016.

[6] R.E. Burkard, S.E Karisch, and F. Rendl. QAPLIB- a quadratic assignment problem library. European Journal of Operational Research, 55:115 - 119, 1991.

[7] R.E. Burkard, S.E. Karisch, and F. Rendl. QAPLIB- a quadratic assignment problem library. Journal of Global Optimization, 10:391-403, 1997.

[8] H. Cambazard and B. O'Sullivan. Propagating the bin packing constraint using linear programming. In Principles and Practice of Constraint Programming - CP 2010, volume 6308 of Lecture Notes in Computer Science, pages 129-136. Springer Berlin Heidelberg, 2010.

[9] A. Caprara, M. Dell'Amico, J.C. Díaz Díaz, M. Iori, and R. Rizzi. Friendly bin packing instances without integer round-up property. Mathematical Programming, 150:5-17, 2014.

[10] E.G. Coffman Jr., J. Csirik, G. Galambos, S. Martello, and D. Vigo. Bin packing approximation algorithms: Survey and classification. In P.M. Pardalos, D.-Z. Du, and R.L. Graham, editors, Handbook of Combinatorial Optimization. Springer New York, 2013.

[11] E.G. Coffman Jr., M.R. Garey, and D.S. Johnson. Approximation algorithms for binpacking - an updated survey. In G. Ausiello, M. Lucentini, and P. Serafini, editors, Algorithm Design for Computer System Design, pages 49-106. Springer Vienna, 1984. 
[12] E.G. Coffman Jr., M.R. Garey, and D.S. Johnson. Approximation algorithms for bin packing: a survey. In D.S. Hochbaum, editor, Approximation algorithms for NP-hard problems, pages 46-93. PWS Publishing Co., 1996.

[13] G. Costa, M. Delorme, M. Iori, E. Malaguti, and S. Martello. A training software for orthogonal packing problems. Technical report, DEI "Guglielmo Marconi", University of Bologna, Italy, 2017.

[14] M. Delorme, M. Iori, and S. Martello. Bin packing and cutting stock problems: Mathematical models and exact algorithms. European Journal of Operational Research, 255:1$20,2016$.

[15] H. Dyckhoff. A new linear programming approach to the cutting stock problem. Operations Research, 29:1092-1104, 1981.

[16] H. Dyckhoff. A typology of cutting and packing problems. European Journal of Operational Research, 44:145-159, 1990.

[17] H. Dyckhoff and U. Finke. Cutting and Packing in Production and Distribution. PhysicaVerlag, Heidelberg, 1992.

[18] E. Falkenauer. A hybrid grouping genetic algorithm for bin packing. Journal of Heuristics, 2:5-30, 1996.

[19] H.A. Friberg. CBLIB 2014: a benchmark library for conic mixed-integer and continuous optimization. Mathematical Programming Computation, 8:191-214, 2016.

[20] M.G. Garey and D.S. Johnson. Computers and intractability. A guide to the theory of NP-completeness. Freeman, New York, 1979.

[21] M.R. Garey and D.S. Johnson. Approximation algorithms for bin-packing problems: A survey. In G. Ausiello and Lucertini, editors, Analysis and Design of Algorithms in Combinatorial Optimization, pages 147-172. Springer Vienna, 1981.

[22] T. Gau and G. Wäscher. CUTGEN1: A problem generator for the standard one-dimensional cutting stock problem. European Journal of Operational Research, 84(3):572-579, 1995.

[23] C. Groër, B. Golden, and E. Wasil. A library of local search heuristics for the vehicle routing problem. Mathematical Programming Computation, 2:79-101, 2010.

[24] T. Gschwind and S. Irnich. Dual inequalities for stabilized column generation revisited. INFORMS Journal on Computing, 28:175-194, 2016.

[25] T. Koch, T. Achterberg, E. Andersen, O. Bastert, T. Berthold, R.E. Bixby, E. Danna, G. Gamrath, A.M. Gleixner, S. Heinz, A. Lodi, H. Mittelmann, T. Ralphs, D. Salvagnin, D.E. Steffy, and K. Wolter. MIPLIB 2010. Mathematical Programming Computation, 3:103, 2011.

[26] J. Lysgaard. CVRPSEP: A package of separation routines for the capacitated vehicle routing problem. Technical report, Aarhus School of Business, Denmark, 2003. 
[27] J. Lysgaard, A.N. Letchford, and R.W. Eglese. A new branch-and-cut algorithm for the capacitated vehicle routing problem. Mathematical Programming, 100:423-445, 2004.

[28] S. Martello and P. Toth. Knapsack Problems: Algorithms and Computer Implementations. John Wiley \& Sons, Chichester, 1990, (available on line at www.or.deis.unibo. it).

[29] M.R. Rao. On the cutting stock problem. Journal of the Computer Society of India, 7:35-39, 1976.

[30] D.M. Ryan and B.A. Foster. An integer programming approach to scheduling. In A. Wren, editor, Computer scheduling of public transport urban passenger vehicle and crew scheduling, pages 269-280. North-Holland, 1981.

[31] J.E. Schoenfield. Fast, exact solution of open bin packing problems without linear programming. Technical report, US Army Space and Missile Defense Command, Huntsville, Alabama, USA, 2002.

[32] A. Scholl, R. Klein, and C. Jürgens. Bison: a fast hybrid procedure for exactly solving the one-dimensional bin packing problem. Computers $\&$ Operations Research, 24:627645, 1997.

[33] P. Schwerin and G. Wäscher. The bin-packing problem: a problem generator and some numerical experiments with FFD packing and MTP. International Transactions in Operational Research, 4:377-389, 1997.

[34] R.M.A. Silva, M.G.C. Resende, P.M. Pardalos, and M.J. Hirsch. A Python/C library for bound-constrained global optimization with continuous GRASP. Optimization Letters, $7(5): 967-984,2013$.

[35] P.E. Sweeney and E.R. Paternoster. Cutting and packing problems: a categorized, application-orientated research bibliography. Journal of the Operational Research Society, 43:691-706, 1992.

[36] E. Uchoa, D. Pecin, A. Pessoa, M. Poggi, T. Vidal, and A. Subramanian. New benchmark instances for the capacitated vehicle routing problem. European Journal of Operational Research, 257:845-858, 2017.

[37] J.M. Valério de Carvalho. Exact solution of bin-packing problems using column generation and branch-and-bound. Annals of Operations Research, 86:629-659, 1999.

[38] J.M. Valério de Carvalho. LP models for bin packing and cutting stock problems. European Journal of Operational Research, 141:253-273, 2002.

[39] G. Wäscher and T. Gau. Heuristics for the integer one-dimensional cutting stock problem: a computational study. Operations-Research-Spektrum, 18:131-144, 1996.

[40] G. Wäscher, H. Haußner, and H. Schumann. An improved typology of cutting and packing problems. European Journal of Operational Research, 183:1109-1130, 2007. 


\section{SPRINGER NATURE Change of authorship request form}

Section 4 Proposed new authorship. Please provide your new authorship list in the order you would like it to appear on the manuscript.

\begin{tabular}{|c|c|c|}
\hline & First name(s) & $\begin{array}{l}\text { Family name (this name will appear in full on the final publication and will be searchable in various abstract and indexing } \\
\text { databases) }\end{array}$ \\
\hline $1^{\text {st }}$ author & Maxence & Delorme \\
\hline $2^{\text {nd }}$ author & Manuel & Iori \\
\hline $3^{\text {rd }}$ author & Silvano & Martello \\
\hline \multicolumn{3}{|l|}{$4^{\text {th }}$ author } \\
\hline \multicolumn{3}{|l|}{$5^{\text {th }}$ author } \\
\hline \multicolumn{3}{|l|}{$6^{\text {th }}$ author } \\
\hline $7^{\text {th }}$ author & & \\
\hline
\end{tabular}

Please use an additional sheet if there are more than 7 authors.

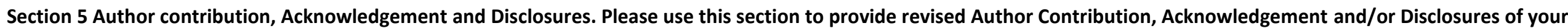

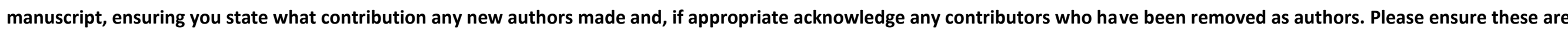
updated in your manuscript.

New Disclosures (potential conflicts of interest, funding, acknowledgements):

New Author Contributions statement:

New Acknowledgement Section: 